\section{SOI: $1.1 /$ TAS DOI: $10.15863 / \mathrm{TAS}$ International Scientific Journal Theoretical \& Applied Science}

\author{
p-ISSN: 2308-4944 (print) e-ISSN: 2409-0085 (online) \\ Year: $2016 \quad$ Issue: $10 \quad$ Volume: 42
}

Published: $30.10 .2016 \quad$ http://T-Science.org
O.A. Aivazian

post graduate student, department "Information design technologies", National Polytechnic University of Odessa ogannes701@gmail.com

SECTION 4. Computer science, computer engineering and automation.

\title{
NON-BINARY CODING METHOD BY SEQUENTIALLY DETERMINED INFORMATIONAL BLOCKS
}

\begin{abstract}
Author presents a model and a method for non-binary coding of information. The system that solves the problem of non-binary coding consists of informational blocks determined in the certain consecutively situated blocks and their interrelated vectors, which form angles relatively to the axis. The paper describes the algorithm of coding and decoding of information using sequence of useful bits and added screening information to the angular relations. Paper considers an example of the encoding operation when we apply a random sequence of non-binary symbols to the input of the encoder in a decimal form. Proposed method supposed for further program and hardware non-binary codes application.

Key words: non-binary coding, angles ratios, sequential informational blocks.

Language: Russian

Citation: Aivazian OA (2016) NON-BINARY CODING METHOD BY SEQUENTIALLY DETERMINED INFORMATIONAL BLOCKS. ISJ Theoretical \& Applied Science, 10 (42): 70-76.

Soi: http://s-o-i.org/1.1/TAS-10-42-16 Doi: crossef http://dx.doi.org/10.15863/TAS.2016.10.42.16

\section{НЕДВОИЧНОЕ КОДИРОВАНИЕ МЕТОДОМ ПОСЛЕДОВАТЕЛЬНО ЗАДАННЫХ ИНФОРМАЦИОННЫХ БЛОКОВ}

Аннотация: Представлень модель и метод недвоичного кодирования информации, где проверочная информация определяется при соотношении последовательно расположенньх блоков и их углов относительно оси. Предложен алгоритм кодирования и декодирования информации в котором задана ограниченной последовательности полезных битов и добавлена проверочная информация за счёт векторов, которые образуют угловые соотношения с осью. Рассматривается режим кодирования с примером работь при подаче на вход кодирующего устройства некоторой последовательности недвоичных символов в десятичном виде. Предложенный метод является основой для дальнейших исследований программного и аппаратного применения недвоччных кодов.
\end{abstract}

Ключевые слова: недвоччное кодирование, угловая мера, последовательность информационных блоков

\section{Введение}

Особенности построения системы кодирования и декодирования и размерность системы счисления могут оказать существенное влияние как на функционирование устройств обработки, так и на их технической реализацию. Реализация в ближайшем будущем многоустойчивых элементов выдвинет на передний край проблему разработки прикладной теории многозначных функций алгебры логики, что следует из [1,2]. Проведённый анализ [3-6] показал, что к настоящему времени нет надёжных способов обнаружения и исправления ошибок в недвоичной реализации, вследствие чего откладывается их аппаратная реализация.
Развитие телекоммуникационных технологий делает чрезвычайно актуальной задачу нахождения новых эффективных путей в реализации методов недвоичного кодирования информации. Так, в работе [7] изложен подход кодирования методом однозначно заданных блоков. При разработке новых алгоритмов основное внимание уделяется эффективности и трудоёмкости алгоритмов кодирования и декодирования. Согласно [6,8] такие алгоритмы необходимы при проектировании экспертных систем, принятии сложных логических решений, аналитическом представлении изображений и их обработке, синтезе и анализе дискретных (абстрактных) автоматов в решении задач 


\begin{tabular}{|c|c|c|c|c|c|c|}
\hline Impact Factor: & $\begin{array}{l}\text { ISRA (India) } \\
\text { ISI (Dubai, UAF } \\
\text { GIF (Australia) } \\
\text { JIF }\end{array}$ & $\begin{array}{l}=1.344 \\
=0.829 \\
=0.564 \\
=1.500\end{array}$ & $\begin{array}{l}\text { SIS (USA) } \\
\text { PИНЦ (Russia) } \\
\text { ESJI (KZ) } \\
\text { SJIF (Morocco) }\end{array}$ & $\begin{array}{l}=0.912 \\
=0.234 \\
=1.042 \\
=2.031\end{array}$ & $\begin{array}{l}\text { ICV (Poland) } \\
\text { PIF (India) } \\
\text { IBI (India) }\end{array}$ & $\begin{array}{l}=6.630 \\
=1.940 \\
=4.260\end{array}$ \\
\hline
\end{tabular}

целочисленной оптимизации, обработки больших многомерных данных, криптографических систем и т.Д.

Целью работы является разработка модели и описание алгоритма недвоичного помехоустойчивого кода, который кодирует информацию, добавляя проверочную часть, затем в процессе декодирования выполняет проверку на возникшие ошибки.

\section{1. Недвоичное представление кода}

В [9] даётся правило, что основополагающими в кодировании информации являются два свойства - свойство избыточности, предполагающая использование избыточных символов для каждого сообщения, и свойство усреднения шума, предполагающего зависимость избыточного символа от нескольких информационных символов.

Модель недвоичного кодирования информации можно представить в виде последовательности информационных блоков (ПИБ), каждый из которых имеет одно независимое состояние. ПИБ порядка $\mathrm{n}$ представляет собой равномерно квадрированный квадрат [10], в котором задана последовательность кодовых символов от 0 до $n^{2}$. В задачах недвоичного кодирования важно построить быстрые алгоритмы кодирования и декодирования при работе с достаточно большими многозначными данными. Предположим, на вход кодирующего устройства будет подаваться кодовые символы длиной L. При поступлении информационных слов необходимо задать некоторую избыточную информацию, за счёт которого будет происходить дальнейшее обнаружение и исправление ошибок. При построении ПИБ каждый блок однозначно определён. Для того чтобы задать проверочную информацию относительно каждого информационного блока (ИБ) целесообразно выделить группы блоков с определёнными признаками. Если спроецировать вектор из точки пересечения блоков $0,1,2,3$ в направлении любых других блоков, на проекции заданного вектора будут находится определённые блоки. На рис. 1 представлен ПИБ размерностью $\mathrm{n}=8$, и спроецирован вектор над блоками $\{1,9,25,49\}$, угловая мера (угол между вектором и осью $O x$ ) $A$ которой равна $45^{\circ}$. Учитывая, что несколько ИБ могут задаваться одним проверочным значением, такой способ добавления избыточной информации позволит построить более быстрые алгоритмы кодирования и декодирования. При этом ИБ, заданные под одним и тем же углом находятся на больших кодовых расстояниях друг от друга, что позволит более эффективно разработать алгоритм декодирования.

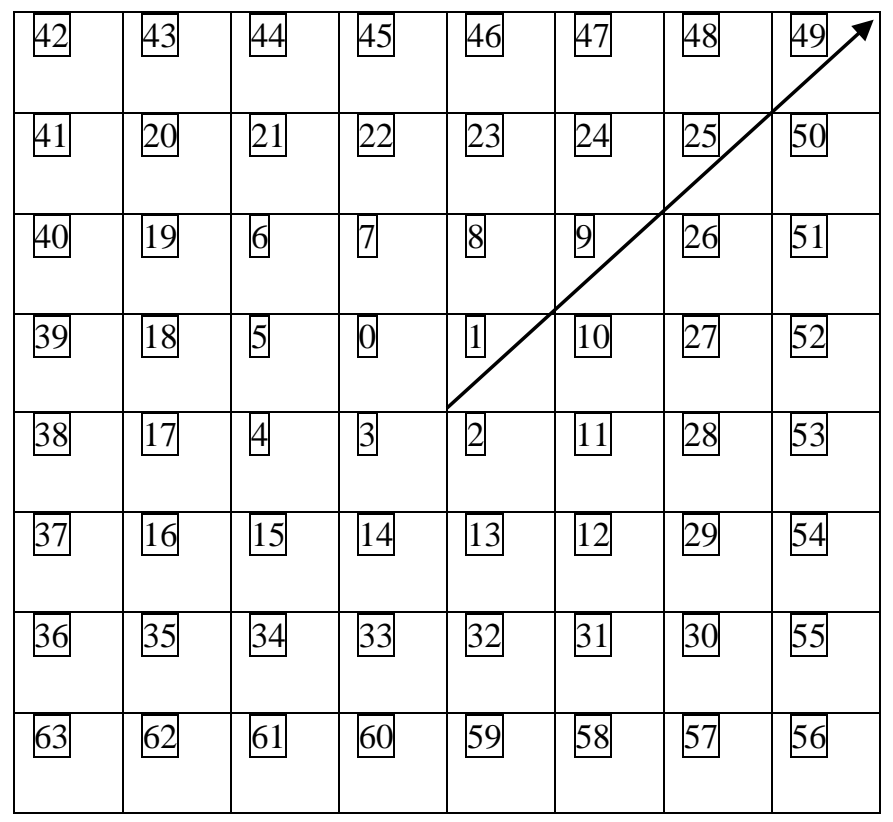

Рисунок 1 - Вектор с $\mathrm{A}=45^{\circ}$ над ПИБ (n=8).

В данном методе вектор задаются над теми информационными блоками (ИБ), соответствующие значения $\mathrm{S}$ которых поданы на вход кодера.

Определение 1. Информационный S-блок - определённый блок информации, принимающий на входе некоторый двоичный код, и преобразующая их в десятичный код с присвоением угловой меры. В обобщённом виде с учётом рис. 1 соотношения $\mathrm{S}-$ блоков и А можно

ISPC Computer technologies in science, 


\begin{tabular}{|c|c|c|c|c|c|c|}
\hline Impact Factor: & $\begin{array}{l}\text { ISRA (India) } \\
\text { ISI (Dubai, UAE } \\
\text { GIF (Australia) } \\
\text { JIF }\end{array}$ & $\begin{array}{l}=1.344 \\
=0.829 \\
=0.564 \\
=1.500\end{array}$ & $\begin{array}{l}\text { SIS (USA) } \\
\text { PИHЦ (Russic } \\
\text { ESJI (KZ) } \\
\text { SJIF (Morocc }\end{array}$ & $\begin{aligned} &= \mathbf{0} .912 \\
&= \mathbf{0} .234 \\
&= \mathbf{1 . 0 4 2} \\
&=\mathbf{2 . 0 3 1}\end{aligned}$ & $\begin{array}{l}\text { ICV (Poland) } \\
\text { PIF (India) } \\
\text { IBI (India) }\end{array}$ & $\begin{array}{l}=6.630 \\
=1.940 \\
=4.260\end{array}$ \\
\hline
\end{tabular}

выразить в виде (1) и (2):

$$
\begin{aligned}
& \forall S_{1}, S_{2}, \ldots, S_{L} \exists A_{i}, \\
& \forall A \ni \min n(S)=1 \\
& \forall A \text { э } \max n(S)=\frac{n}{2},
\end{aligned}
$$

где $\min n(S), \max n(S)$ - минимальное, максимальное количество $S$ соответственно.

Из (1) следует - каждый ИБ обладает своей угловой мерой.

Из (2) следует - каждой А соответствует минимум один $\mathrm{S}$, максимум $-\frac{n}{2} \mathrm{~S}$.

\section{2. Описание метода кодирования}

Для удобства представления кодируемой информации в соответствии с рис. 1 при подаче на вход кодировщика заданную последовательности символов преобразуем в десятичный вид. В случае если на вход кодировщика подаётся двоичная последовательность, то каждый такт m работы кодировщика будет состоять из восьми символов. Тогда максимально возможная последовательность одного такта $m$ будет иметь вид 11111111 , что даёт $255_{10}$. С учётом этого и основываясь на (1), (2) обобщённая модель для кодирования ПИБ с $\mathrm{n}=16$, в котором максимальное количество ИБ составляет 256 описана в виде определения 2.

\section{Определение 2.}

1) Углы $A$ образуют кодовые подмножества в которых элементами являются значения информационных $\mathrm{S}$ - блоков, которые загружены в память кодирующего устройства. В каждое кодовое подмножество входит заданное количество элементов.

2) Минимальное количество ИБ соответствующих некоторому А равно 3 значениям ИБ.

3) Одно и то же $\mathrm{S}$ не повторяется для разных $\mathrm{A}$. Соответствия множеств значений $\mathrm{S}$ для каждого А показаны в (3):

$$
\begin{aligned}
& A=135 \rightarrow\{0,6,20,42,72,110,156,210\} ; A=45 \rightarrow\{1,9,25,49,81,121,169,225\} ; \\
& A=315 \rightarrow\{2,12,30,56,90,132,182,240\} ; A=225 \rightarrow\{3,15,35,63,99,143,195,255\} ; \\
& A=175 \rightarrow\{68,105,150,203\} ; A=170 \rightarrow\{39,151,204\} ; A=165 \rightarrow\{5,18,106\} ; \\
& A=160 \rightarrow\{69,152,205\} ; A=155 \rightarrow\{40,107,206\} ; A=150 \rightarrow\{19,70,108,153,207\} ; \\
& A=145 \rightarrow\{41,71,154,208\} ; A=140 \rightarrow\{109,155,209\} ; A=130 \rightarrow\{73,111,157,211\} ; \\
& A=125 \rightarrow\{21,43,158,212\} ; A=120 \rightarrow\{74,112,159,213\} ; A=115 \rightarrow\{44,113,214\} ; \\
& A=110 \rightarrow\{75,160,215\} ; A=105 \rightarrow\{22,114,161\} ; A=100 \rightarrow\{7,45,216\} ; \\
& A=95 \rightarrow\{76,115,162,217\} ; A=85 \rightarrow\{77,116,163,218\} ; A=80 \rightarrow\{8,46,217\} ; \\
& A=75 \rightarrow\{23,117,164\} ; A=70 \rightarrow\{78,165,220\} ; A=65 \rightarrow\{47,118,221\} ; \\
& A=235 \rightarrow\{34,62,193,253\} ; A=60 \rightarrow\{79,119,166,222\} ; A=55 \rightarrow\{24,48,167,223\} ; \\
& A=50 \rightarrow\{80,120,168,224\} ; A=40 \rightarrow\{122,170,226\} ; A=35 \rightarrow\{50,82,171,227\} ; \\
& A=30 \rightarrow\{26,83,123,172,228\} ; A=25 \rightarrow\{51,124,229\} ; A=20 \rightarrow\{84,173,230\} ; \\
& A=15 \rightarrow\{10,27,125\} ; A=10 \rightarrow\{52,174,231\} ; A=5 \rightarrow\{85 ; 126,175,232\} ; \\
& A=355 \rightarrow\{86,127,176,233\} ; A=350 \rightarrow\{53,177,234\} ; A=345 \rightarrow\{11,28,128\} ; \\
& A=340 \rightarrow\{87,178,235\} ; A=335 \rightarrow\{54,129,236\} ; A=330 \rightarrow\{29,88,130,179,237\} ; \\
& A=325 \rightarrow\{55,89,180,238\} ; A=320 \rightarrow\{131,181,239\} ; A=310 \rightarrow\{91,133,183,241\} ; \\
& A=305 \rightarrow\{31,57,184,242\} ; A=300 \rightarrow\{92,134,185,243\} ; \\
&\{58,135,244\} ; A=290 \rightarrow\{93,186,245\} ; A=285 \rightarrow\{32,136,187\} ; A=280 \rightarrow\{13,59,246\} ; \\
& A=275 \rightarrow\{94,137,188,247\} ; A=265 \rightarrow\{95,138,189,248\} ; A=260 \rightarrow\{14,60,249\} ; \\
& A=255 \rightarrow\{33,139,190\} ; A=250 \rightarrow\{96,191,250\} ; A=245 \rightarrow\{61,140,251\} ;
\end{aligned}
$$




\begin{tabular}{l|lr|ll|ll} 
& ISRA (India) & $=\mathbf{1 . 3 4 4}$ & SIS (USA) & $=\mathbf{0 . 9 1 2}$ & ICV (Poland) & $=\mathbf{6 . 6 3 0}$ \\
Impact Factor: & ISI (Dubai, UAE) $=\mathbf{0 . 8 2 9}$ & PUHЦ (Russia) $=\mathbf{0 . 2 3 4}$ & PIF (India) & $=\mathbf{1 . 9 4 0}$ \\
& GIF (Australia) & $\mathbf{0 . 5 6 4}$ & ESJI (KZ) & $=\mathbf{1 . 0 4 2}$ & IBI (India) & $=\mathbf{4 . 2 6 0}$ \\
& JIF & $=\mathbf{1 . 5 0 0}$ & SJIF (Morocco) & $=\mathbf{2 . 0 3 1}$ & & \\
\hline
\end{tabular}

$$
\begin{gathered}
A=240 \rightarrow\{97,141,192,252\} ; A=235 \rightarrow\{34,62,193,253\} ; A=230 \rightarrow\{98,142,194,254\} ; \\
A=220 \rightarrow\{100,144,196\} ; A=215 \rightarrow\{36,64,145,197\} ; A=210 \rightarrow\{16,65,101,146,198\} ; \\
A=205 \rightarrow\{37,102,199\} ; A=200 \rightarrow\{66,147,200\} ; A=195 \rightarrow\{4,17,103\} ; \\
A=190 \rightarrow\{38,148,201\} ; A=185 \rightarrow\{67,104,149,202\} .
\end{gathered}
$$

4) Учитывая заданные зависимости множеств кодовых символов от их угловых значений, можно вычислить минимальное расстояние между кодовыми блоками в выражении (4):

1. $d_{0}=2 \sin (\pi / 36)=0.174$

2. Алгоритм кодирования

В литературе [11] дано определение избыточности кода, который рассчитывается по формуле (5):

$$
\begin{gathered}
D=k /(k+i), \\
Y_{k}=S_{i 1}+\ldots+S_{p 1}+S_{i 2}+\ldots+S_{p 2}+\ldots+S_{i j}+\ldots+S_{p j}=v_{1}\left(A_{1}\right)+v_{2}\left(A_{2}\right)+\ldots+v_{m}\left(A_{m}\right),
\end{gathered}
$$$$
Y_{1}=v_{1}+v_{2}+v_{3}=320 ;
$$

где $m=1 . .68$, так как всего 68 подмножеств $A$, возможные значения $p=3 \ldots 8$ согласно (3)

Из (6) следует, что при кодировании $k$-тая контрольная сумма $Y$ равна суммам выбранных $S_{\text {и }}$ соответствующим им кодовым подмножествам $A$.

Пример: Пусть по каналу на вход кодирующего устройства подаётся информационное сообщение $u_{1} u_{2} u_{3} u_{4} u_{5} u_{6}$ в виде $\{00000011000001100000111000101010$

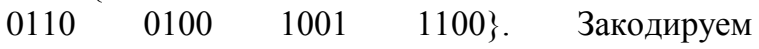
$u_{1} u_{2} u_{3} u_{4} u_{5} u_{6}$ в недвоичное сообщение вида $w_{1} w_{2} \ldots w_{m}$. Каждый недвоичный кодовый символ выражается восемью двоичными, для этого двоичное сообщение делится на блоки по 8 символов в каждом. Основываясь на (3), (6) запишем метод в виде шагов с примером. Шаг 1: с учётом группирования подмножеств (3), формируются недвоичные кодовые символы. Всего задействованы 6 недвоичных символов, которые принадлежат трём подмножествам $A$. Вычислим в (7) суммы для каждого $v_{i}$ и их общую сумму:

$$
\begin{aligned}
& v_{1}: 3+99=102 \\
& v_{2}: 6+42+156=204 \\
& v_{3}: 14
\end{aligned}
$$

Шаг 2: Вычислим в (8) контрольную сумму по выражению (6): где $k-$ количество проверочных единиц измерения информации, $i$ - количество информационных единиц измерения информации.

В представленном методе кодирования избыточность для каждого блока может варьироваться в зависимости от того как расположены ИБ.

Обобщённая формула для контрольной суммы $m$ кодовых слов задана в (6):
Шаг 3: Зададим в (9) количество кодовых символов в каждом $v_{i}$ :

$$
n_{1}=2, n_{2}=3, n_{3}=1 ;
$$

Шаг 4: Сформируем информационное сообщение вида $w_{1} w_{2} \ldots w_{m}$ заданное в виде (10):

$$
w_{i}=v_{i} A_{i} n_{i}, \text { где } i=\overline{1, m}
$$

$n=1$ не добавляется в кодовое слово.

Тогда, в соответствии с примером закодированное сообщение $w_{1} w_{2} w_{3}$ имеет вид:

\begin{tabular}{|l|r|r|r|r|r|r|r|}
\hline 102 & 225 & 2 & 204 & 135 & 3 & 14 & 260 \\
\hline $\mathrm{v}$ & $\mathrm{A}$ & $\mathrm{n}$ & $\mathrm{v}$ & $\mathrm{A}$ & $\mathrm{n}$ & $\mathrm{v}$ & $\mathrm{A}$ \\
\hline
\end{tabular}

Схематически такой код можно отображён в виде рис. 2.

По выражению (5) вычислим избыточность заданного кода в (11):

$$
D=5 /(5+6)=45 \%
$$

Вычислим в (12) максимально и минимально возможные избыточности кода по выражению (5), тогда $D_{\text {Min }}$ получим в случае $n\left(v_{i}\right)=8$, $D_{\text {Max }}$ - в случае $n\left(v_{i}\right)=1$ : 


\begin{tabular}{|c|c|c|c|c|c|c|}
\hline Impact Factor: & $\begin{array}{l}\text { ISRA (India) } \\
\text { ISI (Dubai, UAE } \\
\text { GIF (Australia) } \\
\text { JIF }\end{array}$ & $\begin{array}{l}=1.344 \\
=0.829 \\
=0.564 \\
=1.500\end{array}$ & $\begin{array}{l}\text { SIS (USA) } \\
\text { PИНЦ (Russia) } \\
\text { ESJI (KZ) } \\
\text { SJIF (Morocco }\end{array}$ & $\begin{array}{l}=0.912 \\
=0.234 \\
=1.042 \\
=\mathbf{2 . 0 3 1}\end{array}$ & $\begin{array}{l}\text { ICV (Poland) } \\
\text { PIF (India) } \\
\text { IBI (India) }\end{array}$ & $\begin{array}{l}=6.630 \\
=1.940 \\
=4.260\end{array}$ \\
\hline
\end{tabular}

$$
\begin{aligned}
& D_{\text {Min }}=2 / 10=20 \% \\
& D_{\text {Max }}=1 / 2=50 \%
\end{aligned}
$$

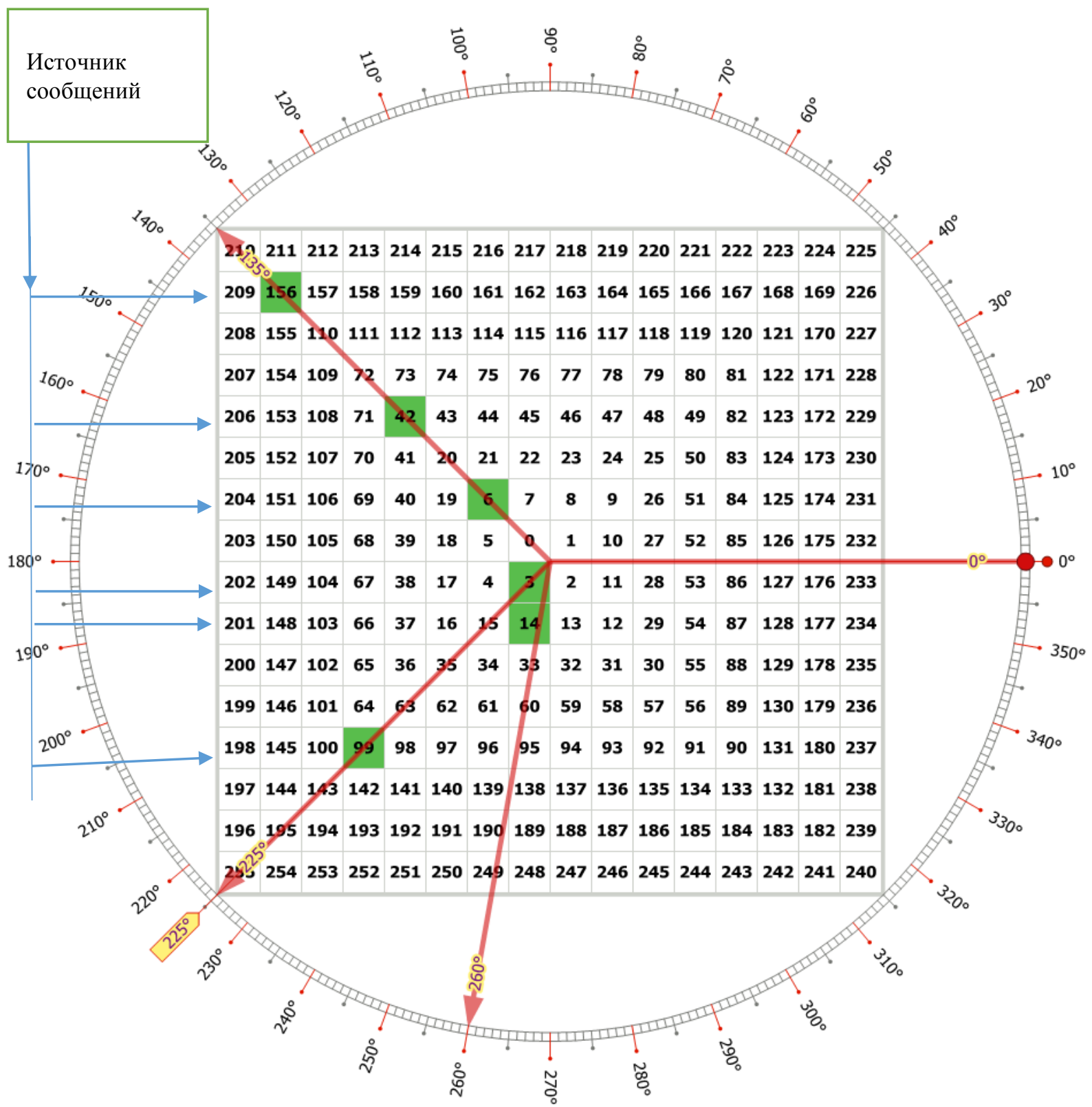

Рисунок 2 - ПИБ (256) с обозначением множества кодируемых $S$ и соответствующим им углам

3. Декодирование и исправление ошибок Алгоритм декодирования предполагает вычисление каждого $v_{i}$ из полученного сообщения $w_{1} w_{2} \ldots w_{m}$. Таким образом, декодирование сводится к задаче о сумме подмножеств. [12,13]. Вычисление искомых $S_{i}$ uз $v_{i}$ представляет собой задачу о сумме подмножеств, для решения которой используется множество алгоритмов. С учётом того что скорее всего декодируемое сообщение будет содержать ошибки вычислительные эксперименты, проведённые на ЭВМ позволили установить, что наиболее оптимальным по надёжности является решение с помощью метода brute force, который подробно описан в [12]. С учётом того что каждое подмножество $A$ содержит от 3 до 8 элементов, как показано в (3), удаётся однозначно находить решение. 


\begin{tabular}{|c|c|c|c|c|c|c|}
\hline Impact Factor: & $\begin{array}{l}\text { ISRA (India) } \\
\text { ISI (Dubai, UAE } \\
\text { GIF (Australia) } \\
\text { JIF }\end{array}$ & $\begin{array}{l}=1.344 \\
=0.829 \\
=0.564 \\
=1.500\end{array}$ & $\begin{array}{l}\text { SIS (USA) } \\
\text { PИНЦ (Russia) } \\
\text { ESJI (KZ) } \\
\text { SJIF (Morocco) }\end{array}$ & $\begin{array}{l}=0.912 \\
=0.234 \\
=1.042 \\
=\mathbf{2 . 0 3 1}\end{array}$ & $\begin{array}{l}\text { ICV (Poland } \\
\text { PIF (India) } \\
\text { IBI (India) }\end{array}$ & $\begin{array}{l}=6.630 \\
=1.940 \\
=4.260\end{array}$ \\
\hline
\end{tabular}

Рассмотрим необходимое условие точного исправления ошибок. Тогда отклонение $\beta$ от закодированной суммы $v_{i}$ должно быть меньше минимального элемента $S$ из некоторого подмножества $A_{i}$. Таким образом, порог исправления ошибок $\beta$ задаётся неравенством (13):

$$
\beta<\min S_{i}\left(A_{i}\right),
$$

Опишем метод декодирования с внесением ошибок удовлетворяющих неравенству (13) в виде шагов. Шаг 1. Декодер получает сообщение вида $w_{1} w_{2}, \ldots, w_{m}$, как задано в конечном Шаге 4 алгоритма кодирования. Шаг 2. Выразим внесение ошибки в $v_{i}$, в результате чего получим $t_{i}$ в (14):

$$
v_{i} \pm \beta_{i}=t_{i},
$$

В качестве примера основываясь на (7) и (13) внесём ошибки $\beta=1$ или 2 в $v_{1}$ и промоделируем на ЭВМ средствами программирования процесс декодирования. Выразим результат в (15):

$$
t_{1}=3+99+2=104 \Rightarrow 3+99
$$

$$
t_{1}=3+99+1=103 \Rightarrow 3+99
$$

Шаг 3. Из декодированных кодовых слов $v_{i}$ можно обратно получить сообщение в двоичном виде $u_{i}$, каждое из которых выражено восемью двоичными.

\section{Заключение}

Предложен метод недвоичного помехоустойчивого кодирования с представлением информации в виде последовательно заданных информационных блоков. Представлен простой и алгоритм кодирования с расчётом избыточности, где результат кодирования - сумма элементов из одного кодового подмножества. Описан метод быстрого декодирования, основывающийся на задаче о сумме подмножеств. Внесённые ошибки позволили установить, что в соответствии со способом декодирования, максимальный порог ошибки равен минимальному элементу из подмножества. Алгебраическое представление кода и его модернизация являются предметом дальнейших исследований.

Предложенный метод может быть использован как для повышения эффективности разработки недвоичных кодов, так и для тестирования в криптографии, аппаратной и дальнейшей программной реализации.

\section{References:}

1. Kuharev GA (1990) Algorithms and systological processors for many-valued data processing / G.A. Kuharev, V.P. Shmerko, Y. N. Zaytseva Minsk: Navuka i technika, 1990

2. Korolev AI (2002) Codes and impairments steady devices coding / A.I. Korolev - Minsk: 2002 - pp. 286

3. Blahut R (1986) Theory and practice of error control codes / R. Blahut - Moscow: Mir, 1986 $-576 \mathrm{p}$.

4. Verner M (2004) Information and Codes / M. Verner - Moscow : Tekhnosfera, 2004 - 286 p.

5. MacWilliams FJ (1979) Theory of ErrorCorrecting Codes / F.J. MacWilliams, N.J.A. Sloane. - Moscow: Svjaz', 1979. — pp. 745.
6. Sklar B (2003) Digital communications fundamentals and applications / B. Sklar Moscow: Vilyams, $2003-1104$ p.

7. Aivazian O (2016) Non-binary codes complex modeling provided in the squares form and rotation vector / O.A. Aivazian, Y. D. Ivanov: Proceedings of modern information and electronic technologies - Odessa, 2016

8. Proakis J (2000) Digital Communication / J. Proakis, M. Salehi - M.: Radio i svjaz', 2000 - 800 p. - ISBN 5-256-01434-X.

9. Clark G (1987) Jr. / Error-Correction coding for digital communications / G. C. Clark, Jr., J. B. Cain - Moscow: Radio i svjaz', 1987. — 392 p.

10. Honsberger R (2015) Squaring the square [Electronic Resource] / University of Waterloo, Faculty of Mathematics, - 2015 


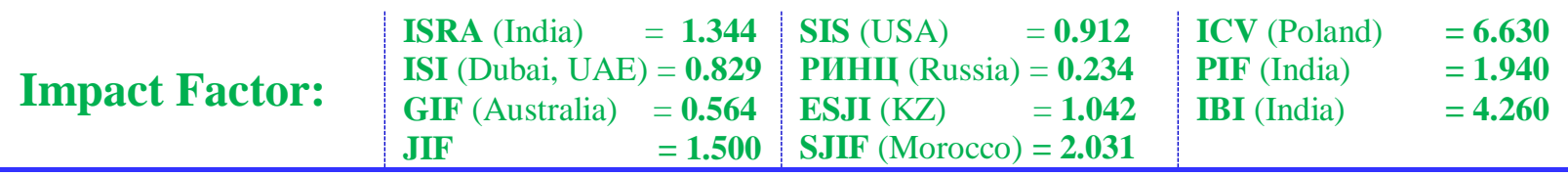

http://www.math.uwaterloo.ca/navigation/ideas/

Leiserson, Ronald L. Rivest: Introduction to articles/honsberger2/index.shtml

11. Piterson W (1976) Error-correcting codes / W. W. Peterson, E. J. Weldon - M.: Mir, 1976 $583 \mathrm{p}$.

12. Cormen T (2009) Chapter 35.5: The subset-sum problem / Thomas H. Cormen, Charles E. Algorithms, 3rd Edition. - MIT Press, 2009. ISBN 978-0-262-03384-8.

13. Garey M (1979) Computers and Intractability: A Guide to the Theory of NP-Completeness. / Michael R. Garey and David S. Johnson W.H. Freeman, 1979. — ISBN 0-7167-1045-5. 